\title{
Light exotic hadrons at BESIII
}

\author{
Jianbin Jiao ${ }^{1, a}$, on behalf of BESIII Collaboration \\ ${ }^{1}$ Shandong University, Jinan, China
}

\begin{abstract}
We review the recent results for the light exotic hadron states, which are obtained using the data recorded with the BESIII detector at the BEPCII $e^{+} e^{-}$collider in Beijing, China.
\end{abstract}

\section{Introduction}

The quark model is a valid effective classification scheme in particle physics, which organized the large number of light hadrons successfully and verified by the experiments in the past few decades. But beyond the conventional hadrons, which are the combinations of 3-quarks or quark-antiquark, the quantum chromodynamics (QCD) also allows the existence of the exotic hadron states, e. g., multiquark states, glueballs and hybrids. In the last decade, a lot of experiment groups devoted to study the exotic hadron states and a few of candidates have been reported.

BESIII detector is a general-purpose magnetic spectrometer at the double-ring $e^{+} e^{-}$collider BEPCII and is used for the study of physics in the $\tau$-charm energy region [1]. It has a geometrical acceptance of $93 \%$ of $4 \pi$ solid angle and consists of four main subsystems: a helium-based multilayer drift chamber (MDC), a plastic scintillator time-of-flight (TOF) system, a CsI (Tl) electromagnetic calorimeter (EMC) and a resistive plate muon chamber system. The first three subdetectors are enclosed in a superconducting solenoidal magnet with a $1.0 \mathrm{~T}$ magnetic field. More details of the detector are described in Ref. [2].

The BESIII experiment has successfully accumulated the world's largest 1,300 million $J / \psi$ events [3] and 450 million $\psi(3686)$ events [4], which offers a clean laboratory to study light exotic hadron states below the open charm threshold. In this talk, we review the recent results about the research of light exotic states, which are made of only light quarks, from BESIII experiment based on these data samples.

\section{States near $1.85 \mathrm{GeV}$}

The $\mathrm{X}(1835)$ state was first observed in the decay of $J / \psi \rightarrow \gamma \eta^{\prime} \pi^{+} \pi^{-}$by BESII experiment [5]. Using the 225 million $J / \psi$ events taken with BESIII detector in 2009, this state is confirmed by BESIII experiment in the same decay mode with an improved statistical significance of more than $20 \sigma$ [6], which is shown in Fig. 1(a). It is worth to mentioned that the resonances near $2.1 \mathrm{GeV} / c^{2}$ and 2.4 $\mathrm{GeV} / c^{2}$, the $X(2120)$ and $X(2370)$, are observed in the $\pi^{+} \pi^{-} \eta^{\prime}$ mass spectrum with more than $5 \sigma$ in this

\footnotetext{
ae-mail: jiaojb@sdu.edu.cn
} 
measurement too. In another measurement of $J / \psi \rightarrow \gamma K_{s} K_{s} \eta$ decay [7], the structure near $1.85 \mathrm{GeV}$ is also studied based on the full statistics of $J / \psi$ events. Figure 1(b) shows a clear structure around $1.85 \mathrm{GeV} / c^{2}$ in the $K_{s} K_{s} \eta$ mass spectrum. The mass, width and spin parity are determined, by a partial wave analysis (PWA) performed to the events with $M_{K_{s} K_{s}}<1.1 \mathrm{GeV} / c^{2}$ and $M_{K_{s} K_{s} \eta}<2.8 \mathrm{GeV} / c^{2}$, to be $1844 \pm 9(\text { stat })_{-25}^{+16}$ (syst) $\mathrm{MeV} / c^{2}, 192_{-17}^{+20}(\text { stat })_{-43}^{+62}($ syst $) \mathrm{MeV} / c^{2}$ and $J^{P C}=0^{-+}$, respectively. These results are consistent with the BESIII results in the measurement of $J / \psi \rightarrow \gamma \eta^{\prime} \pi^{+} \pi^{-}$decay [6].
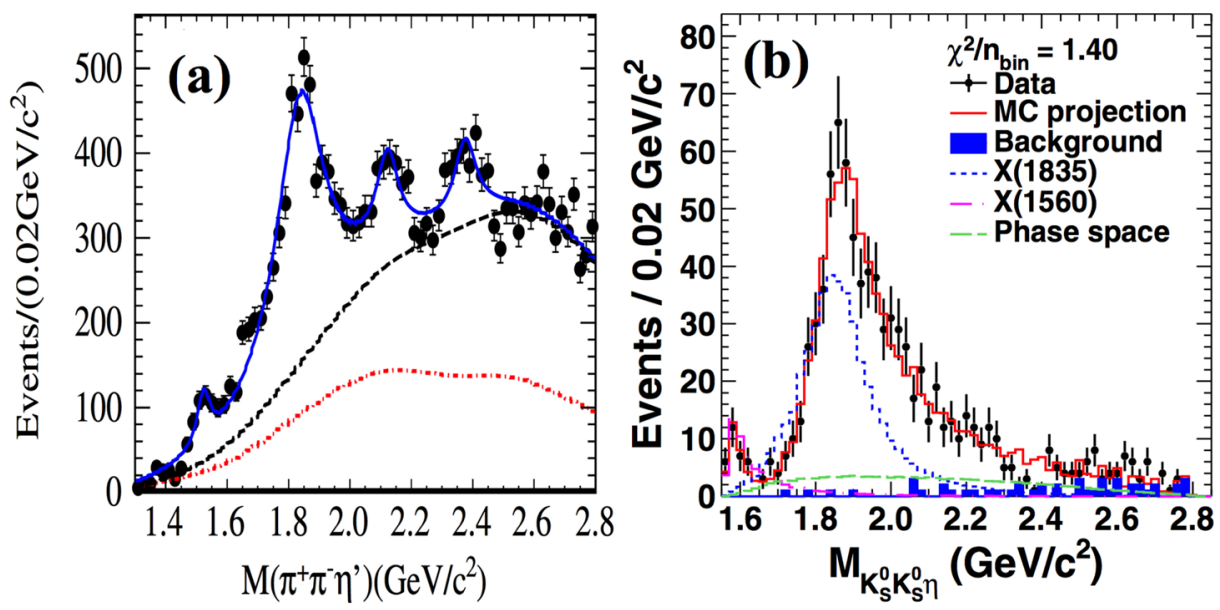

Figure 1. (a) Fit the $\pi^{+} \pi^{-} \eta^{\prime}$ mass spectrum with four resonances. (b) Fit of the $K_{s} K_{s} \eta$ mass spectrum for events with the requirement of $M_{K_{s} K_{s}}<1.1 \mathrm{GeV} / c^{2}$ and PWA projections.

Actually, BESIII Collaboration observed similar structures near $1.85 \mathrm{GeV}$ in various decay modes recently [8-11]. Figure 2 shows the enhancement near $p \bar{p}$ mass threshold in the $p \bar{p}$ mass spectrum and the comparison to different BESIII results for masses and widths. Most of the masses are in agreement with each other and close to the $p \bar{p}$ mass threshold, while the widths are significantly different. Therefore, based on these experimental results, one cannot drawn a conclusion about the connections between all these structures. Further studies with more decay modes are essential to understand the nature of the structures around $1.85 \mathrm{GeV}$.

In the latest BESIII result published in 2016 [12], the structure near $1.85 \mathrm{GeV}$ is studied with the decay of $J / \psi \rightarrow \gamma \eta^{\prime} \pi^{+} \pi^{-}$, using the 1090 million $J / \psi$ events collected by BESIII detector in 2012. Two typical models are used to characterize the $\eta^{\prime} \pi^{+} \pi^{-}$anomalous line shape around $1.85 \mathrm{GeV}$. One model assumes the state couples with the $p \bar{p}$ and the distortion reflects the opening of the $p \bar{p}$ decay channel, another one describes the distortion as the interference between the $X(1835)$ and another resonance with a mass close to the $p \bar{p}$ mass threshold. Figure 3 shows the fit of the $\eta^{\prime} \pi^{+} \pi^{-}$mass spectrum based on these two models, respectively. Both fits show almost equally good agreement with data, we cannot tell which model is better, while either one supports the existence of a $p \bar{p}$ moleculelike state or bound state with a significance greater than $7 \sigma$.

\section{Study of $Y(2175)$ state}

The study of strangeonium mesons is important since they are a bridge between the light $\mathrm{u}, \mathrm{d}$ quarks and the heavy c, b quarks and also helps to identify the exotic states which decay to the same final 

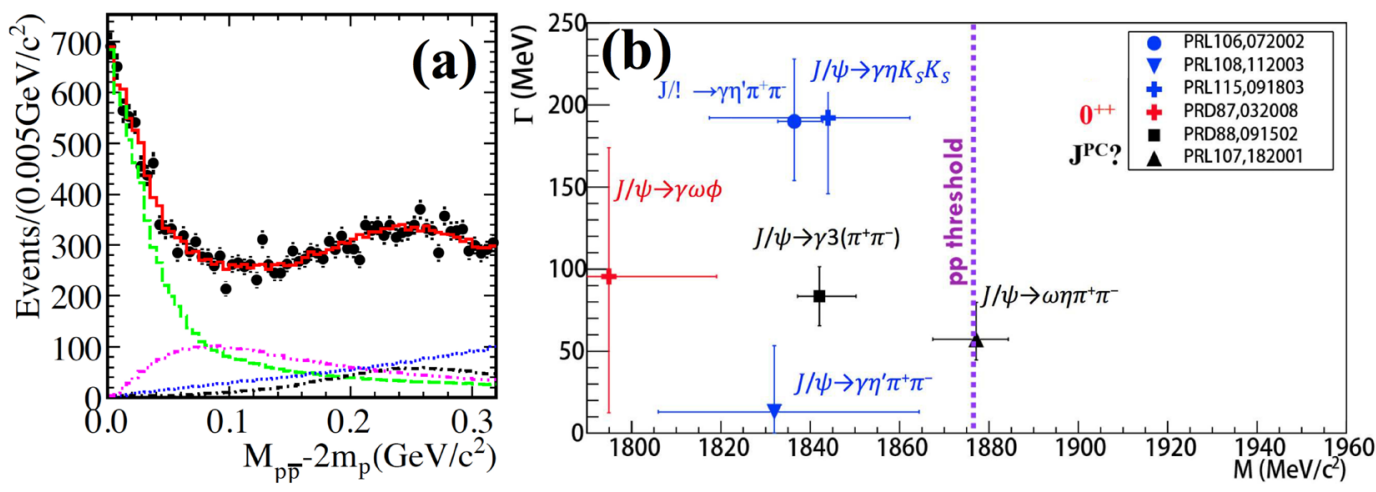

Figure 2. (a) Enhancement near $p \bar{p}$ mass threshold; (b) comparison to different BESIII results for masses and widths.
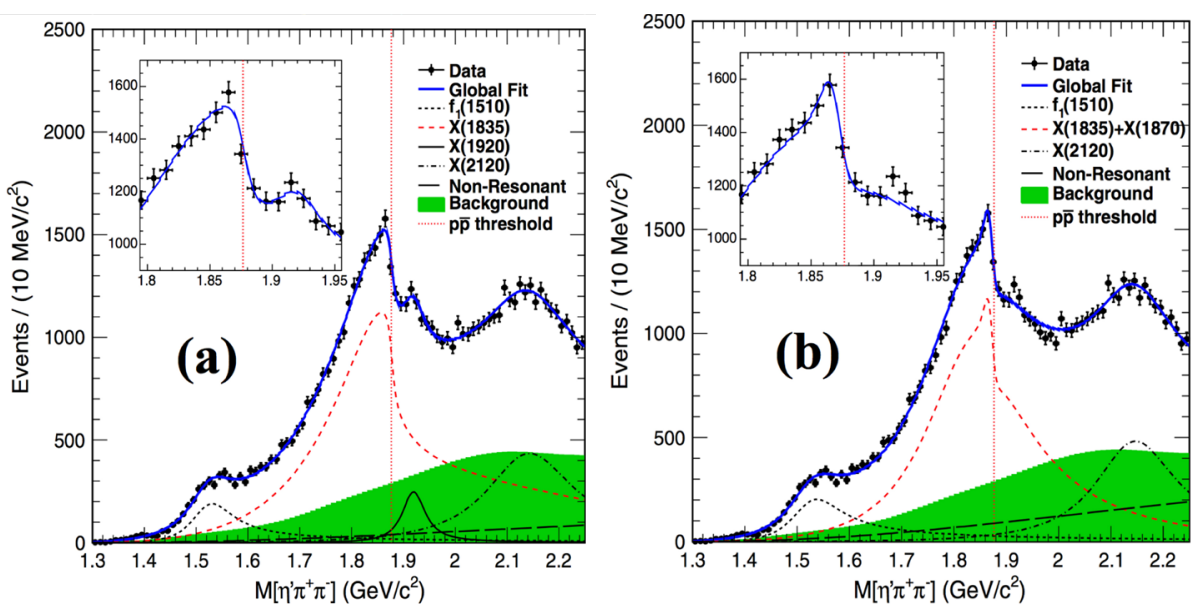

Figure 3. Fits of the state near $1.85 \mathrm{GeV}$ in the $\eta^{\prime} \pi^{+} \pi^{-}$mass spectrum by assuming that (a) the state couples to the $p \bar{p}$ and the distortion above the $p \bar{p}$ threshold reflects the opening of the $p \bar{p}$ decay channel; (b) the distortion is caused by the interference between the $X(1835)$ and another resonance with mass close to the $p \bar{p}$ mass threshold.

states. In the prediction of relativistic quark model, the strangeonium $(s \bar{s})$ states have a spectroscopy similar to the heavy quarkonia. But most of the members in strangeonium spectrum have not been observed. The experimental measurement of strangeonium mesons is subject to the small $s \bar{s}$ states production among hadrons and large width of the states. The high statistics of data sample collected with the BESIII detector provide a good laboratory to study the strangeonium and help to distinguish the exotics from the conventional mesons.

The $Y(2175)$ state, also referred to as the $\phi(2170)$, is speculated to be an $s$-quark counterpart to the $Y(4260)$ since they have similar production mechanism and decay patterns. The nature of this state stimulates further experimental measurements. We present the results of a study for the 
$J / \psi \rightarrow \eta \phi \pi^{+} \pi^{-}$decay [13] using a sample of 225 million $J / \psi$ events taken with the BESIII detector in 2009. The $Y(2175)$ state was observed in the mass spectrum of $\phi f_{0}(980)$ with a statistical significance of greater than $10 \sigma$ and shown in Fig. 4. The correlated mass and width are determined to be $2200 \pm$ $6($ stat $) \pm 5$ (syst) $\mathrm{MeV} / c^{2}$ and $104 \pm 15($ stat $) \pm 15$ (syst) $\mathrm{MeV}$, respectively, and the product branching fraction for the decays $J / \psi \rightarrow \eta Y(2175), Y(2175) \rightarrow \phi f_{0}(980), f_{0}(980) \rightarrow \pi^{+} \pi^{-}$is measured to be $(1.20 \pm 0.14($ stat $) \pm 0.37$ (syst $)) \times 10^{-4}$. The results are consistent with those of previous experiments within errors.

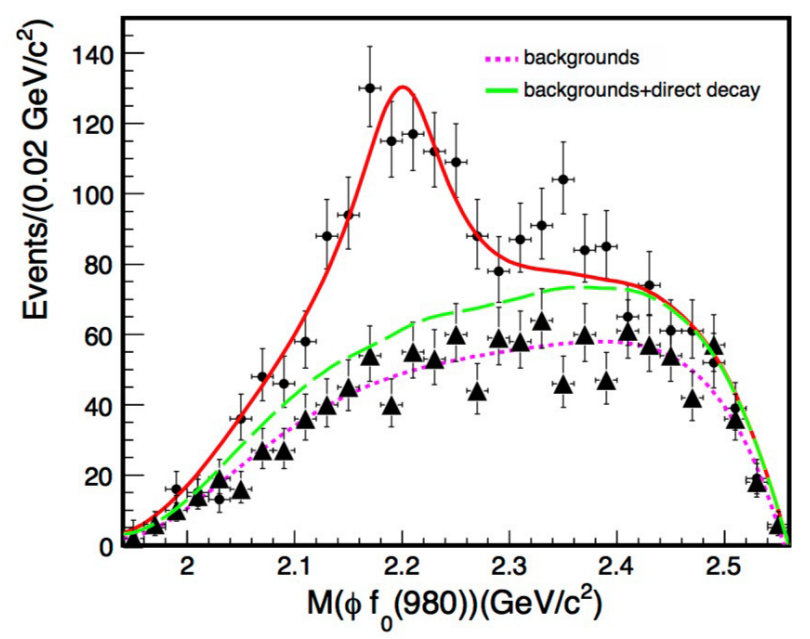

Figure 4. Result of the fit of the $\phi f_{0}(980)$ invariant mass distribution.

\section{Search for glueballs}

The existence of glueballs has been predicted by QCD, According to the theoretical calculation of Lattice QCD, the mass of the ground state glueball, with a spin parity equal to $0^{++}$, should be around 1,600 to $1,700 \mathrm{MeV} / c^{2}$, while the mass for the first excited state, $2^{++}$should be about $2,300 \mathrm{MeV} / \mathrm{c}^{2}$. The $0^{-+}$state and exotic glueballs (with non- $q \bar{q}$ quantum numbers such as $0^{--}, 0^{+-}, 1^{-+}, 2^{+-}$, etc.) are expected above $2 \mathrm{GeV}$. These mass ranges are accessible for the BESIII experiment.

The amplitude analysis for the two pseudoscalar spectrum in radiative $J / \psi$ decays is relative simple. Conservation rules, such as the conservation of parity in strong and electromagnetic interactions and the conservation of angular momentum, restrict the quantum numbers of pseudoscalarpseudoscalar pair. Only the amplitudes with even angular momentum, positive parity and charge conjugation quantum numbers are possible $\left(J^{P C}=0^{++}, 2^{++}, 4^{++}\right.$, etc. $)$. Initial studies suggest that only the $0^{++}$and $2^{++}$amplitudes are significant in radiative $J / \psi$ decays to $\eta \eta$ or $\pi^{0} \pi^{0}$.

The PWA results for the decay of $J / \psi \rightarrow \gamma \eta \eta$ [14], which is shown in Fig. 5(a), indicate that the scalar contributions are mainly from $f_{0}(1500), f_{0}(1700)$ and $f_{0}(2100)$ in the $\eta \eta$ mass spectrum, the $f_{0}(1700)$ and $f_{0}(2100)$ states are the dominant scalars. No evident contributions from $f_{0}(1370)$ and $f_{0}(1790)$ are found. The recent prediction of lattice QCD for the production rate of the pure gauge scalar glueball in $J / \psi$ radiative decays [15] are found to be consistent with the $f_{0}(1700)$ production rate in $J / \psi$ radiative decay in this analysis, which suggests that the $f_{0}(1700)$ has a large overlap with 
the glueball than other candidates (e.g., $f_{0}(1500)$ ). The contribution of tensor components in $\eta \eta$ mass spectrum are from $f_{2}^{\prime}(1525), f_{2}(1810)$ and $f_{2}(2340)$, the first one is the dominant tensor. There is a component lying in the mass region from $1.8 \mathrm{GeV} / c^{2}$ to $2 \mathrm{GeV} / c^{2}$ with a statistical significance of $6.6 \sigma$, but we cannot distinguish $f_{2}(1810)$ from $f_{2}(1910)$ or $f_{2}(1950)$ in current statistics. The PWA requires a strong contribution from $f_{2}(2340)$, although the possibility of $f_{2}(2300)$ cannot be ruled out. There is no evidence of $f_{J}(2220)$ found in this analysis.

We present another analysis for the decay of $J / \psi \rightarrow \gamma \pi^{0} \pi^{0}$ with the model-independent PWA applied [16]. The $M_{\pi^{0} \pi^{0}}$ mass spectrum is shown in Fig. 5(b). The PWA results indicate that the scalar contributions are mainly from the structures $\sigma(600), f_{0}(1370), f_{0}(1500), f_{0}(1710)$ and $f_{0}(2020)$, while the $f_{2}(1270)$ is dominant tensor component in the $M_{\pi^{0} \pi^{0}}$ mass spectrum.
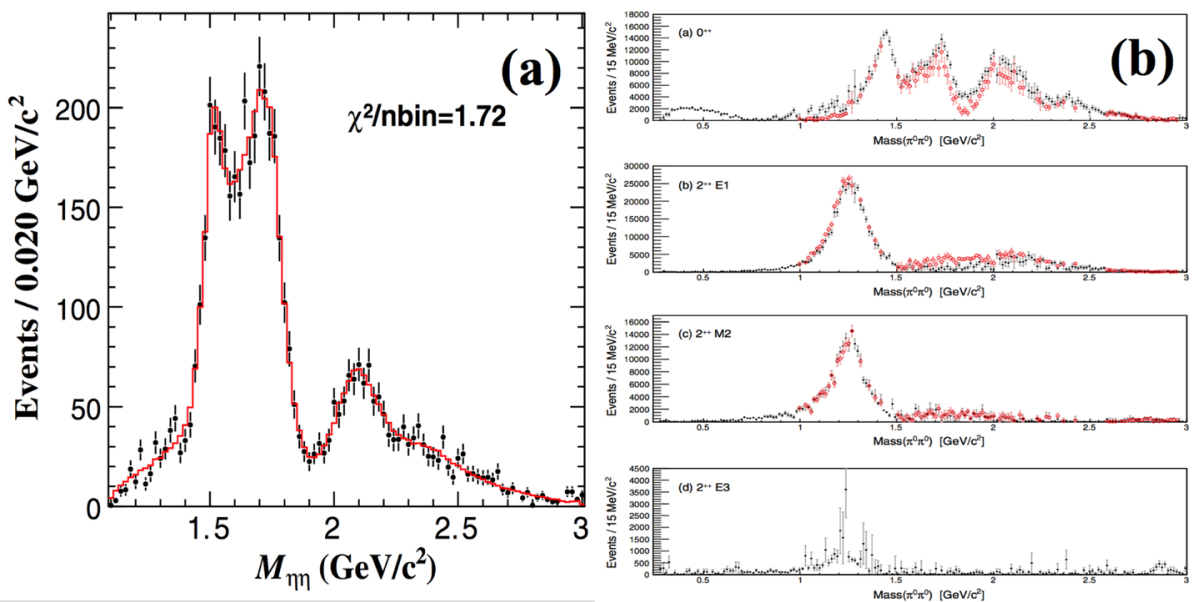

Figure 5. (a) Comparisons between data and PWA fit projections for $\eta \eta$ mass spectrum; (b) Intensities for various amplitude components as a function of $M_{\pi^{0} \pi^{0}}$ for the nominal results.

Recently, a PWA of the $J / \psi \rightarrow \gamma \phi \phi$ decay is applied to study the intermediate states [17]. Figure 6 show the mass spectrum of $\phi \phi$ and the intensities of individual $J^{P C}$ components, respectively. The existence of $\eta(2225)$ is confirmed and its resonance parameters are measured in this analysis. Two additional pseudoscalar states, $\eta(2100)$ with a mass of $2050_{-19-23}^{+30+75} \mathrm{MeV} / c^{2}$ and a width of $250_{-30-164}^{+36+181}$ $\mathrm{MeV}$ and $X(2500)$ with a mass of $2470_{-19-23}^{+15+101} \mathrm{MeV} / \mathrm{c}^{2}$ and a width of $230_{-35-33}^{+64+56} \mathrm{MeV}$, are observed. Three tensor states, $f_{2}(2010), f_{2}(2300)$ and $f_{2}(2340)$ are reported in this analysis too. The production rate of the pure gauge tensor glueball in $J / \psi$ radiative decays, which is predicted by Lattice QCD [15], is compatible with the large production rate of the $f_{2}(2340)$ in this analysis and in $J / \psi \rightarrow \gamma \eta \eta$ [14].

Although the low-lying glueballs are expected to exist in an accessible energy ranges for current colliders, they are still extremely difficult to be identified since they mix with the ordinary meson states. A few of pseudoscalar states have been observed in various radiative decays of $J / \psi$ at BESIII experiment. To map out the pseudoscalar states and search for glueballs, the systematic studies for more radiative decay modes of $J / \psi$ are needed. 

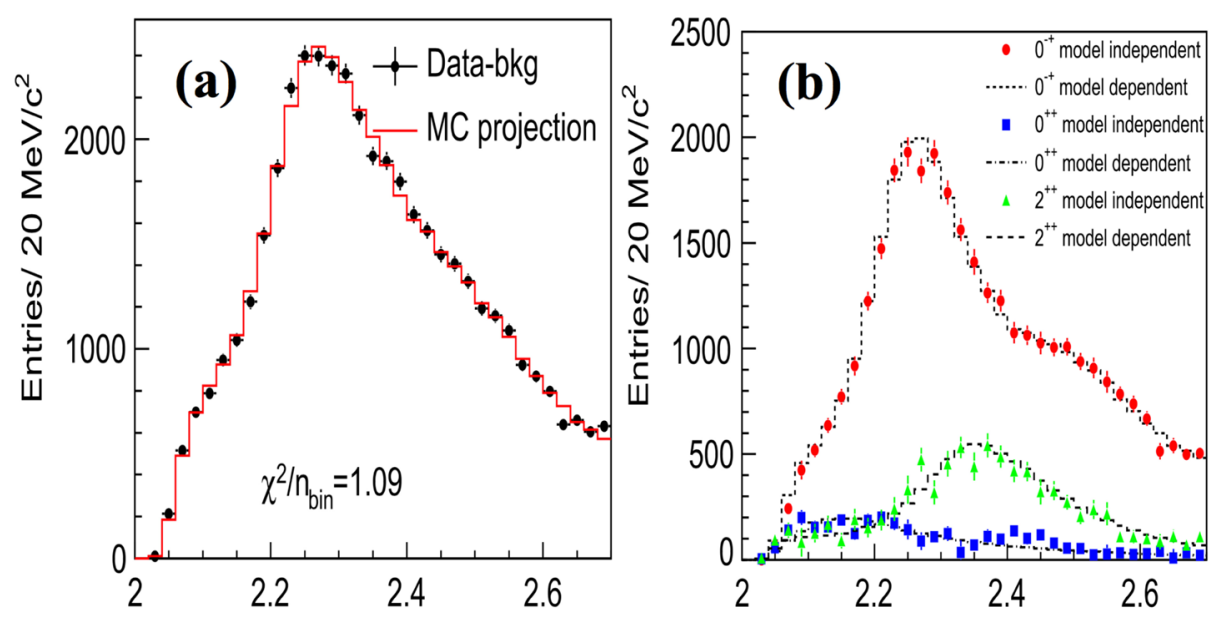

Figure 6. (a) Comparisons between data and PWA fit projections for $\phi \phi$ mass spectrum; (b) Intensities for various amplitude components in $\phi \phi$ mass spectrum.

\section{Search for exotics}

States with exotic spin parities, such as $0^{--}, 0^{+-}, 1^{-+}, 2^{+-}$and $3^{-+}$, etc., are beyond the naive quark model and easily distinguished from ordinary states. But till now, the production rate and dynamics of them are not well understood.

The charmonium state, like the $\chi_{c 1}$ with a spin parity equal to $1^{++}$, which is easily produced in the rediative decay of $\psi(3686)$ at $e^{+} e^{-}$colliders, provide suitable laboratory for studying the production of exotic mesons with $J^{P C}=1^{-+}$in its decay mode of $\chi_{c 1} \rightarrow \eta \pi \pi$.

We present results from the amplitude analysis of the full decay $\psi(3686) \rightarrow \gamma \chi_{c 1}, \chi_{c 1} \rightarrow \eta \pi^{+} \pi^{-}$ with high purity [14]. The $\eta \pi$ mass spectrum is shown in Fig. 7 and the possible exotic meson production in the $\eta \pi$ invariant mass region from $1.4 \mathrm{GeV} / c^{2}$ to $2.0 \mathrm{GeV} / c^{2}$ is examined. Table 1 lists the fraction contributions and significances of the three $J^{P C}=1^{-+}$candidates, $\pi_{1}(1400), \pi_{1}(1600)$ and $\pi_{1}$ (2015). Two possible orbital-momentum configurations for an exotic amplitude are the $S$-wave and $D$-wave, and the significance of each is tested individually. The $\pi_{1}(1400)$, with a significance of $3.5 \sigma$ and fractional contribution less than $0.6 \%$, is the most significant one of the three exotic state candidates. This represents a weak evidence for the existence of $\pi_{1}(1400)$ because the significance of this state becomes $<3 \sigma$ when parameters of other amplitudes are varied. Our conclusion is that there is no significant evidence for an exotic $\eta \pi$ structure in the $\chi_{c 1} \rightarrow \eta \pi^{+} \pi^{-}$decay and we determine the upper limits on the respective branching fractions of $\pi_{1}$ decays.

Table 1. List of fractional intensities, significances of amplitudes in the base-line fit and upper limits for the productions of $\pi_{1}$ candidates

\begin{tabular}{cccc}
\hline \hline Decay & Fractional intensity [\%] & Significance $[\sigma]$ & Production $\left[10^{-3}\right]$ \\
\hline$\pi_{1}(1400)^{+} \pi^{-}$ & $0.58 \pm 0.20$ & 3.5 & $<0.046$ \\
$\pi_{1}(1600)^{+} \pi^{-}$ & $0.11 \pm 0.10$ & 1.3 & $<0.015$ \\
$\pi_{1}(2015)^{+} \pi^{-}$ & $0.06 \pm 0.03$ & 2.6 & $<0.008$ \\
\hline \hline
\end{tabular}




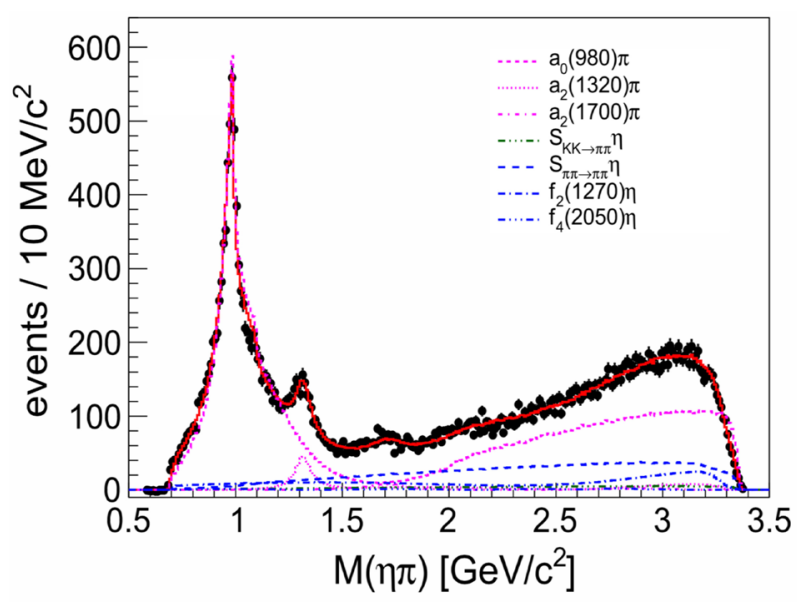

Figure 7. Projection in the $\eta \pi$ invariant mass from data, compared with the base-line fit and corresponding amplitudes.

\section{Summary}

Besides the confirmation of $X(1835)$ in $J / \psi$ rediative decay, more structures around $1.85 \mathrm{GeV}$ are observed in various decay modes at BESIII experiment and show connections between each other according to the comparison of mass, width and spin parity. The study of strangeonium state, $Y(2175)$, is also presented. For the searching of glueballs, we presents the PWA results for the rediative decays of $J / \psi$ to $\eta \eta, \pi^{0} \pi^{0}$ and $\phi \phi$. A few of pseudoscalar states, with masses lying in glueball mass region which are predicted by Lattice QCD, are studied. More rediative decay modes of $J / \psi$ are essential in the systematic studies in the future. We also present the results for the searching of exotic mesons with $J^{P C}=1^{-+}$in the decay $\chi_{c 1} \rightarrow \eta \pi \pi$, while the amplitude analysis shows no significant evidence for the existence of the corresponding $\pi_{1}$ states in this decay.

Based on the world's largest statistics of $J / \psi$ and $\psi(3686)$ events accumulated at the BESIII detector, the recent results for the study of the light exotic hadron states at BESIII experiment are presented in this talk, more interesting results about this topic will be reported by BESIII Collaboration soon.

\section{Acknowledgement}

The BESIII collaboration thanks the staff of BEPCII and the IHEP computing center for their strong support. This work is supported in part by National Key Basic Research Program of China under Contract Nos. 2015CB856700; National Natural Science Foundation of China (NSFC) under Contracts Nos. 11235011, 11335008, 11425524, 11625523, 11635010; the Chinese Academy of Sciences (CAS) Large-Scale Scientific Facility Program; the CAS Center for Excellence in Particle Physics (CCEPP); Joint Large-Scale Scientific Facility Funds of the NSFC and CAS under Contracts Nos. 11179007, U1232106, U1232201, U1332201, U1732102; CAS Key Research Program of Frontier Sciences under Contracts Nos. QYZDJ-SSW-SLH003, QYZDJ-SSW-SLH040; 100 Talents Program of CAS; National 1000 Talents Program of China; INPAC and Shanghai Key Laboratory for Particle Physics and Cosmology; German Research Foundation DFG under Contracts Nos. Collaborative 
Research Center CRC 1044, FOR 2359; Istituto Nazionale di Fisica Nucleare, Italy; Koninklijke Nederlandse Akademie van Wetenschappen (KNAW) under Contract No. 530-4CDP03; Ministry of Development of Turkey under Contract No. DPT2006K-120470; National Natural Science Foundation of China (NSFC) under Contracts Nos. 11505034, 11575077; National Science and Technology fund; The Swedish Research Council; U. S. Department of Energy under Contracts Nos. DE-FG0205ER41374, DE-SC-0010118, DE-SC-0010504, DE-SC-0012069; University of Groningen (RuG) and the Helmholtzzentrum fuer Schwerionenforschung GmbH (GSI), Darmstadt; WCU Program of National Research Foundation of Korea under Contract No. R32-2008-000-10155-0.

\section{References}

[1] D. M. Asner et al., Int. J. Mod. Phys. A 24, 499 (2009).

[2] M. Ablikim et al. (BESIII Collaboration), Nucl. Instrum. Methods Phys. Res., Sect. A 614, 345 (2010).

[3] M. Ablikim et al. (BESIII Collaboration), Determination of the number of $J / \psi$ events with $J / \psi \rightarrow$ inclusive decays, Chin. Phys. C 36, 915 (2012); M. Ablikim et al. (BESIII Collaboration), Chin. Phys. C (to be published).

[4] M. Ablikim et al. (BESIII Collaboration), Determination of the number of $\psi(3686)$ events at BESIII, Chin. Phys. C 37, 063001 (2013); The total number of $\psi(3686)$ events is obtained based on the same method. The preliminary number is determined to be $447.9 \times 10^{6}$ with uncertainties of $0.65 \%$.

[5] M. Ablikim et al. (BESIII Collaboration), Phys. Rev. Lett. 95, 262001 (2005).

[6] M. Ablikim et al. (BESIII Collaboration), Phys. Rev. Lett. 106, 072002 (2011).

[7] M. Ablikim et al. (BESIII Collaboration), Phys. Rev. Lett. 115, 091803 (2015).

[8] M. Ablikim et al. (BESIII Collaboration), Phys. Rev. Lett. 107, 182001 (2011).

[9] M. Ablikim et al. (BESIII Collaboration), Phys. Rev. Lett. 108, 112003 (2012).

[10] M. Ablikim et al. (BESIII Collaboration), Phys. Rev. D 87, 032008 (2013).

[11] M. Ablikim et al. (BESIII Collaboration), Phys. Rev. D 88, 091502 (2013).

[12] M. Ablikim et al. (BESIII Collaboration), Phys. Rev. Lett. 117, 042002 (2016).

[13] M. Ablikim et al. (BESIII Collaboration), Phys. Rev. D 91, 052017 (2015).

[14] M. Ablikim et al. (BESIII Collaboration), Phys. Rev. D 87, 092009 (2013).

[15] L. C. Gui et al., Phys. Rev. Lett. 110, 021601 (2013).

[16] M. Ablikim et al. (BESIII Collaboration), Phys. Rev. D 92, 052003 (2015).

[17] M. Ablikim et al. (BESIII Collaboration), Phys. Rev. D 93, 112011 (2016).

[18] M. Ablikim et al. (BESIII Collaboration), Phys. Rev. D 95, 032002 (2017). 